\title{
Nuevos registros de Cephaloleia (Coleoptera: Chrysomelidae) plaga de la palma de aceite en Colombia
}

\author{
New records of Cephaloleia (Coleoptera: Chrysomelidae) pest of oil palm in Colombia \\ CARLOS ENRIQUE BARRIOS-TRILLERAS ${ }^{1}$; NATALIA JULIETH CASTILLO-VILLARRAGA ${ }^{2}$; \\ ALEX ENRIQUE BUSTILLO-PARDEY ${ }^{3}$; CHARLES L. STAINES ${ }^{4}$
}

\begin{abstract}
${ }^{1}$ Ing. Agrónomo, Corporación Centro de Investigación en Palma de Aceite, Cenipalma, Bogotá, Colombia, cbarrios@cenipalma.org, https://orcid.org/00000001-9536-6053. ${ }^{2}$ Ing. Agrónomo, Corporación Centro de Investigación en Palma de Aceite, Cenipalma, Bogotá, Colombia, ncastillo@cenipalma.org, https://orcid.org/0000-0001-7042-0543. ${ }^{3}$ Ing. Agrónomo, Ph. D., Corporación Centro de Investigación en Palma de Aceite, Cenipalma, Bogotá, Colombia, abustillo@cenipalma.org, https://orcid.org/0000-0003-4375-4913. ${ }^{4}$ Department of Entomology, National Museum of Natural History, Smithsonian Institution, Washington DC, EE. UU, stainesc@si.edu, https://orcid.org/0000-0002-7411-1024.
\end{abstract}

\begin{abstract}
Autor para correspondencia: Carlos Enrique Barrios-Trilleras, Ing. Agrónomo, Corporación Centro de Investigación en Palma de Aceite, Cenipalma, Bogotá, Colombia, cbarrios@ cenipalma.org, https://orcid.org/0000-00019536-6053.
\end{abstract}

Citación sugerida / Suggested citation: BARRIOS-TRILLERAS, C. E; CASTILLOVILLARRAGA, N. J.; BUSTILLO-PARDEY, A. E.; STAINES, C. L. 2020. Nuevos registros de Cephaloleia (Coleoptera: Chrysomelidae) plaga de la palma de aceite en Colombia. Revista Colombiana de Entomología 2020, 46 (1): e6863. https://doi.org/10.25100/socolen. v46i1.6863

Recibido: 31-ago-2018

Aceptado: 24-jul-2019

Publicado: 16-jul-2020

Revista Colombiana de Entomología

ISSN (Impreso): 0120-0488

ISSN (En línea): 2665-4385

http://revistacolombianaentomologia.univalle.edu.co/

Open access

Publicadores / Publishers:

Sociedad Colombiana de Entomología

SOCOLEN (Bogotá, D. C., Colombia)

http://www.socolen.org.co

Universidad del Valle (Cali, Colombia)

http://www.univalle.edu.co/

(C) 2020 Sociedad Colombiana de Entomología - SOCOLEN y Universidad del Valle - Univalle
Resumen: Se confirmó la identidad taxonómica de Cephaloleia vagelineata y se registra por primera vez en Colombia la especie Cephaloleia depressa y que causa daños en plantaciones de palma de aceite. También, se ofrece un reconocimiento morfológico de los adultos de ambas especies para facilitar su identificación.

Palabras clave: Cephaloleia depressa, Cephaloleia vagelineata, Elaeis guineensis, Neotrópico.

Abstract: The taxonomic identity of Cephaloleia vagelineata was confirmed and the species Cephaloleia depressa is reported for the first time in Colombia and causing damage in oil palm plantations. Adult morphological descriptions of both species are provided to facilitate their identification.

Keywords: Cephaloleia depressa, Cephaloleia vagelineata, Elaeis guineensis, Neotropic.

\section{Introducción}

El género Cephaloleia Chevrolat, 1836 (Coleoptera: Chrysomelidae) tiene distribución Neotropical e incluye 214 especies registradas desde México hasta Argentina (Staines 1996, 1998; Schmitt y Frank 2013; Staines y García-Robledo 2014). Las especies de este género se hospedan en plantas de las familias Arecaceae y Zingiberaceae (McKenna y Farrell 2005; Staines 2008; García-Robledo et al. 2010).

Urueta (1972) observó por primera vez lesiones en hojas jóvenes de palma de aceite Elaeis guineensis Jacquin (Arecales: Arecaceae) en la región de Urabá (Antioquia, Colombia) e identificó al insecto causante del daño como Cephaloleia aff. vagelineata. Posteriormente, otra especie de este género fue observada en la región de San Carlos de Guaroa, Meta (Aldana et al. 2010) sin determinar su identidad taxonómica. Por lo anterior, el objetivo de esta investigación fue conocer la identidad taxonómica de las especies del género Cephaloleia presentes en plantaciones de palma de aceite en Colombia y describir la morfología externa de los adultos.

\section{Materiales y métodos}

Los especímenes de Cephaloleia fueron recolectados en plantaciones de palma de aceite (E. guineensis e híbridos interespecíficos $\mathrm{O}$ x G) en los departamentos de Antioquia, Bolívar, Casanare, Cesar, Córdoba, Magdalena, Meta, Nariño, Norte de Santander y Santander (Colombia). Los adultos de los especímenes colectados fueron conservados en viales de vidrio con alcohol al $75 \%$, etiquetados con la información del lugar de colecta. Los especímenes fueron trasladados al Laboratorio de Entomología de Cenipalma, en el Campo Experimental Palmar de la Sierra, municipio de Zona Bananera (Magdalena). Se tomaron fotografías detalladas de los diferentes caracteres morfológicos utilizando un estereoscopio Olympus SZ-61, acoplado a una cámara de microfotografía de 14 MP y fueron procesadas con el software Toupview $®$. Esta información fue enviada al Departamento de Entomología del Museo Nacional de Historia Natural del Instituto Smithsoniano en Washington, donde se realizó la 
identificación de las especies con la clave propuesta por Staines y García-Robledo (2014). Para conocer las diferencias entre los especímenes recolectados, se tomaron medidas de longitud y ancho de 50 ejemplares adultos, longitud de los élitros y antenas de las especies identificadas. Los datos se analizaron usando estadística descriptiva (promedio \pm desviación estándar) y se comparó con información registrada de referencias científicas para conocer la distribución geográfica de estas especies y hábitos.

\section{Resultados}

Se recolectaron 326 ejemplares de Cephaloleia vagelineata Pic, 1926 y 62 de Cephaloleia depressa Baly, 1858 (Coleoptera: Chrysomelidae). La especie $C$. vagelineata se encontró causando lesiones en plantaciones de palma de aceite E. guineensis e híbrido en las zonas palmeras de los departamentos de Antioquia, Bolívar, Cesar, Córdoba, Magdalena y Santander, mientras que $C$. depressa solo se encontró en plantaciones de palma en el departamento del Meta (Tabla 1). Los especímenes examinados se conservaron en el Centro de Colecciones Biológicas de la Universidad del Magdalena [CBUMAG].

\section{Cephaloleia vagelineata Pic, 1926}

Material examinado (en parte). Cephaloleia vagelineata. 25ㅇ, 12 $\overbrace{}^{\circ}$. COLOMBIA. Magdalena, Zona Bananera, $10^{\circ} 39^{\prime} 44.16^{\prime \prime} \mathrm{N} 74^{\circ} 11^{\prime} 54.58^{\prime \prime} \mathrm{O}, 30 \mathrm{~m}$. Captura manual, 08ene-2016. Castillo, N. [CBUMAG].

Reconocimiento. Los adultos (Fig. 1A) son de color negro y tienen una marca blanca en forma de "Y" sobre los élitros. La hembra es más grande que el macho y tiene una longitud de 4,8 $\pm 0,3 \mathrm{~mm}$ desde el clípeo hasta el último segmento abdominal y un ancho de $1,4 \pm 0,1$ en la parte media del pronoto, mientras que el macho posee una longitud de 4,2 $\pm 0,3 \mathrm{~mm}$ y un ancho de 1,2 $\pm 0,1$. Las antenas son filiformes, miden 1,4 $\pm 0,04 \mathrm{~mm}$ y presentan nueve artejos antenales, Las patas miden 2,6 \pm $0,2 \mathrm{~mm}$, los tarsos presentan vellosidades color amarillo. En la parte ventral, la sutura entre los segmentos abdominales $1 \mathrm{y}$ 2 no se ven bien definidas (Fig. 1B).

\section{Cephaloleia depressa Baly, 1858}

Material examinado (en parte). Cephaloleia depressa. $9 \circ$, 9ð. COLOMBIA. Meta, Acacías, 358'24,2”N 73³3'48,6”O, 498 m. Captura manual, 15-jul-2016. Aldana, R. [CBUMAG].

Reconocimiento. Los adultos son de color negro (Fig. 2), su cuerpo es aplanado. Los machos son de menor tamaño que las hembras pues miden 4,4 $\pm 0,3 \mathrm{~mm}$ de longitud desde el clípeo hasta el último segmento abdominal y 2,1 $\pm 0,1 \mathrm{~mm}$ de ancho en la parte media del pronoto, mientras que las hembras miden 5,2 $\pm 0,4 \mathrm{~mm}$ de longitud y 2,4 $\pm 0,3 \mathrm{~mm}$ de ancho. Las antenas filiformes con 10 artejos antenales y miden 1,7 $\pm 0,1 \mathrm{~mm}$. El pronoto tiene borde aserrado y de color negro con borde marrón. El escutelo es triangular; los élitros tienen una longitud de $3,1 \pm 0,2 \mathrm{~mm}$ y $1,3 \pm 0,2 \mathrm{~mm}$ de ancho, con márgenes lisos y redondeados en la parte apical. Los élitros y el pronoto presentan impresiones en forma de puntos. Las patas son delgadas, la tibia y los tarsos exhiben una franja de pelos amarillos ubicados en el margen interior y longitud total de las patas 3,2 $\pm 0,3 \mathrm{~mm}$.

\section{Discusión}

El conocimiento de la morfología externa de los insectos, especialmente del orden Coleoptera, permite identificar especímenes usando caracteres diagnósticos para familias,

Tabla 1. Registros de Cephaloleia vagelineata y C. depressa en palma de aceite en Colombia.

\begin{tabular}{|c|c|c|c|c|c|}
\hline Departamento & Municipio & Latitud (N) & Longitud (O) & Especie registrada & No. Especímenes \\
\hline Antioquia & Carepa & $7^{\circ} 38^{\prime} 20.66^{\prime \prime}$ & 76039"11.64" & C. vagelineata & 23 \\
\hline Bolívar & María la baja & $9^{\circ} 55^{\prime} 49.2378^{\prime \prime}$ & $75^{\circ} 19^{\prime} 38.9712^{\prime \prime}$ & C. vagelineata & 21 \\
\hline Bolívar & San Pablo & $7^{\circ} 31^{\prime} 31,2^{\prime \prime}$ & $73^{\circ} 56^{\prime} 12,7^{\prime \prime}$ & C. vagelineata & 11 \\
\hline Cesar & Codazzi & $9^{\circ} 55^{\prime} 28.99^{\prime \prime}$ & $73^{\circ} 18^{\prime} 2.99^{\prime \prime}$ & C. vagelineata & 32 \\
\hline Cesar & El Copey & $10^{\circ} 6^{\prime} 12.30^{\prime \prime}$ & $74^{\circ} 0{ }^{\prime} 52.60^{\prime \prime}$ & C. vagelineata & 28 \\
\hline Córdoba & Montería & $8^{\circ} 24^{\prime} 29.88^{\prime \prime}$ & $75^{\circ} 58^{\prime} 15.8052^{\prime \prime}$ & C. vagelineata & 22 \\
\hline Magdalena & Aracataca & $10^{\circ} 33^{\prime} 1.90^{\prime \prime}$ & $74^{\circ} 11^{\prime} 30.73^{\prime \prime}$ & C. vagelineata & 71 \\
\hline Magdalena & Zona Bananera & $10^{\circ} 39^{\prime} 44.16^{\prime \prime}$ & $74^{\circ} 11^{\prime} 54.58^{\prime \prime}$ & C. vagelineata & 76 \\
\hline Meta & Acacías & $3^{\circ} 58^{\prime} 24,2^{\prime \prime}$ & $73^{\circ} 33^{\prime} 48,6^{\prime \prime}$ & C. depressa & 30 \\
\hline Meta & Acacías & $3^{\circ} 58^{\prime} 31,9^{\prime \prime}$ & $73^{\circ} 35^{\prime} 54,4^{\prime \prime}$ & C. depressa & 7 \\
\hline Meta & San Carlos de Guaroa & $3^{\circ} 45^{\prime} 52,0^{\prime \prime}$ & $73^{\circ} 23^{\prime} 11,0^{\prime \prime}$ & C. depressa & 5 \\
\hline Meta & San Isidro de Chichimene & $3^{\circ} 52^{\prime} 37,0^{\prime \prime}$ & $73^{\circ} 40^{\prime} 11,5^{\prime \prime}$ & C. depressa & 15 \\
\hline Meta & San Carlos de Guaroa & $3^{\circ} 53^{\prime} 07^{\prime \prime}$ & $73^{\circ} 20^{\prime} 06^{\prime \prime}$ & C. depressa & 5 \\
\hline Santander & Puerto Wilches & $7^{\circ} 14^{\prime} 260^{\prime \prime}$ & $73^{\circ} 53^{\prime} 822^{\prime \prime}$ & C. vagelineata & 8 \\
\hline Santander & Puerto Wilches & $7^{\circ} 20^{\prime} 56.7^{\prime \prime}$ & $73^{\circ} 48^{\prime} 19.6$ ' & C. vagelineata & 10 \\
\hline Santander & Puerto Wilches & $7^{\circ} 24^{\prime} 10.7^{\prime \prime}$ & $73^{\circ} 47^{\prime} 01.4^{\prime \prime}$ & C. vagelineata & 7 \\
\hline Santander & Puerto Wilches & $7^{\circ} 15^{\prime} 01.9^{\prime \prime}$ & $73^{\circ} 43^{\prime} 23.3^{\prime \prime}$ & C. vagelineata & 7 \\
\hline Santander & Rionegro & $7^{\circ} 36^{\prime} 2,5^{\prime \prime}$ & $73^{\circ} 40^{\prime} 24^{\prime \prime}$ & C. vagelineata & 5 \\
\hline Santander & Sabana de Torres & $7^{\circ} 18^{\prime} 24,8^{\prime \prime}$ & $73^{\circ} 37^{\prime} 10,8^{\prime \prime}$ & C. vagelineata & 5 \\
\hline
\end{tabular}


A

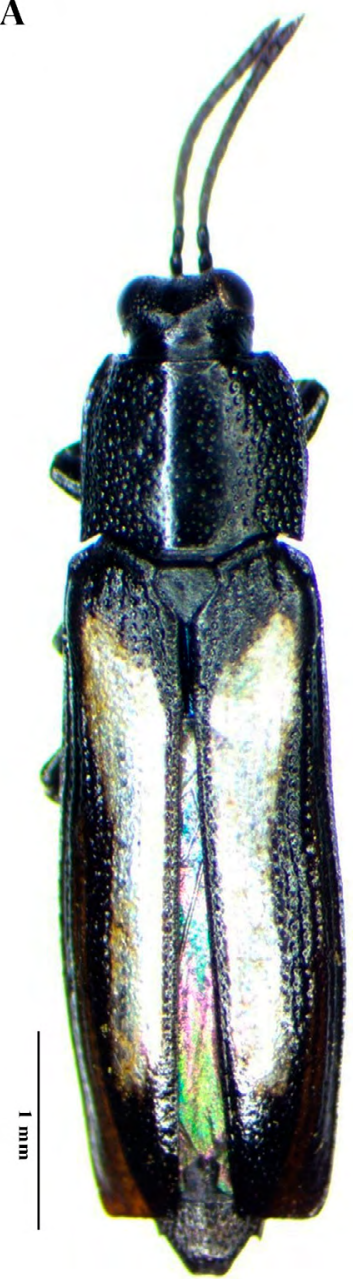

B

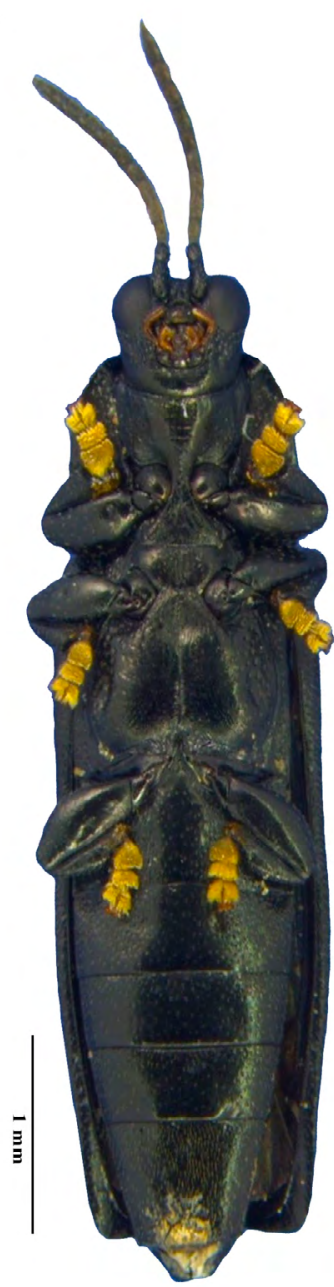

Figura 1. Adulto de adulto de Cephaloleia vagelineata colectado en palma de aceite. A. Vista dorsal. B. Vista ventral. Foto: Carlos Barrios.

género y especie (Chapman 1998; Martínez et al. 2013). La descripción de los adultos, utilizando los caracteres morfológicos aquí expuestos para $C$. vagelineata y $C$. depressa coincide con lo registrado en la literatura para ambas especies (Urueta 1972; 1975; Genty et al. 1978; Staines y GarcíaRobledo 2014) confirmando la identidad de estas especies y el registro por primera vez de $C$. depressa en Colombia.

Los adultos de ambas especies se ubican en las hojas jóvenes y flechas de la palma de aceite, alimentándose de estas y causando un raspado en los foliolos (Urueta 1975; Genty et al. 1978). En ocasiones, se dejan caer de las hojas cuando son perturbados. La hembra deposita los huevos de manera individual sobre las bases peciolares y el raquis de las hojas jóvenes (Urueta 1972). Después de la eclosión, las larvas migran a las bases peciolares e incluso se pueden encontrar en el cogollo de la palma, donde se alimentan causando raspaduras superficiales. $C$. vagelineata se encuentra distribuido en el continente americano desde México hasta Brasil y se ha registrado causando daños en Astrocaryum chonta, Cocos nucifera y Elaeis oleifera (Genty et al. 1978; Staines et al. 2014). Mientras que $C$. depressa solo se había registrado en Brasil y Ecuador en palma de aceite (Mariau 2001; Staines y García-Robledo 2014), por lo que los registros indicados en este estudio constituyen el primer registro de esta especie en Colombia.

A

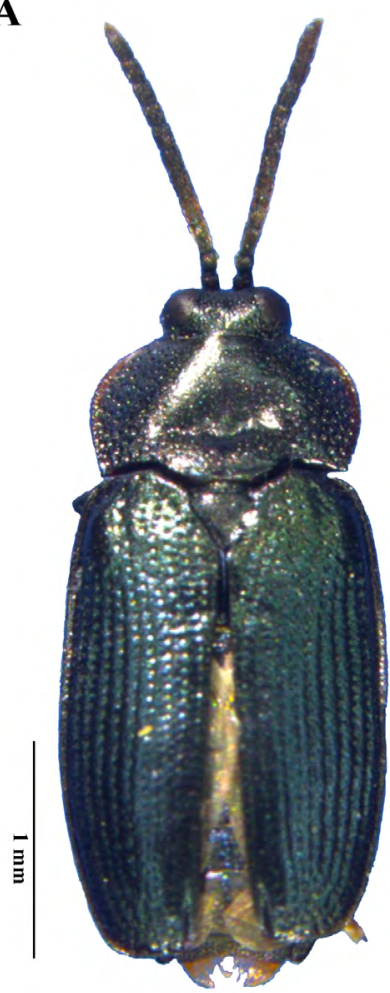

B

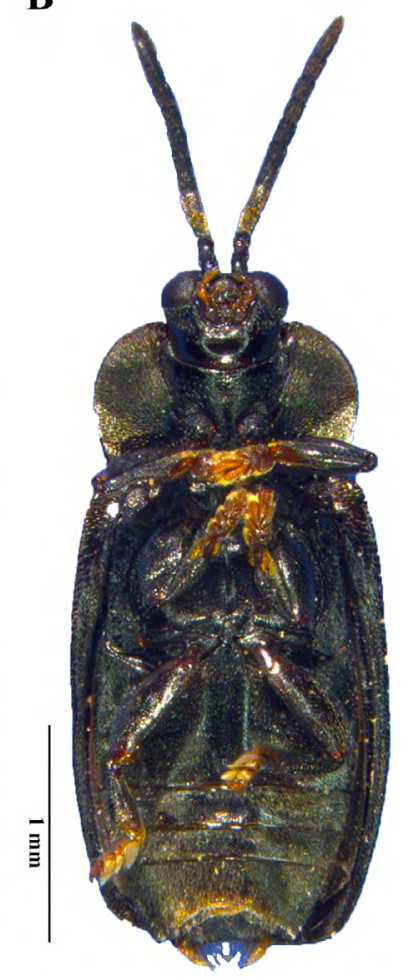

Figura 2. Adulto de adulto de Cephaloleia depressa colectado en palma de aceite. A. Vista dorsal. B. Vista ventral. Foto: Carlos Barrios.

\section{Agradecimientos}

Los autores agradecen al Fondo de Fomento Palmero administrado por Fedepalma y al núcleo Extractora el Roble S.A.S por el apoyo financiero. A los ingenieros Nelson Caicedo, Adalberto Méndez, Luis Guillermo Montes, Carlos Sendoya y Rosa Aldana por su apoyo en el desarrollo de este estudio.

\section{Literatura citada}

ALDANA, R. C.; ALDANA, J. A.; CALVACHE, H.; FRANCO, P. N. 2010. Manual de plagas de la palma de aceite en Colombia. Cuarta edición. SENA - Cenipalma. Bogotá (Colombia), 67 p.

CHAPMAN, R. F. 1998. The insects: structure and function, 4th ed. Cambridge University Press, Cambridge, MA. 962 p.

GARCÍA-ROBLEDO, C.; HORVITZ, C. C.; STAINES, C. L. 2010. Larval morphology, development, and notes on the natural history of Cephaloleia «rolled-leaf» beetles (Coleoptera: Chrysomelidae: Cassidinae). Zootaxa 2610 (1): 50-68. https:// doi.org/10.11646/zootaxa.2610.1.3

GENTY, P.; DESMIER DE CHENON, R.; MORIN, J. P. 1978. Las plagas de la palma aceitera en América Latina. Oleagineux (Francia) 33 (7): 326 - 420

MARIAU, D. 2001. The fauna of oil palm and coconut. Insect and mite pests and their natural enemies. CIRAD. Montpellier, Francia, 249 p.

MARTÍNEZ, L.; PLATA, A.; ZANUNCIO, J.; LEITE, G.; SERRAO, J. 2013. Morphology and morphometry of Demotispa neivai (Coleoptera: Chrysomelidae) adults. Annals of the Entomological Society of America 106 (2):164-169. https://doi. org/10.1603/AN12013

McKENNA, D; FARRELL, B. 2005. Molecular phylogenetics and evolution of host plant use in the Neotropical rolled leaf 
'hispine' beetle genus Cephaloleia (Chevrolat) (Chrysomelidae: Cassidinae). Molecular Phylogenetics and Evolution 37 (1): 117131. https://doi.org/10.1016/j.ympev.2005.06.011

SCHMITT, M.; FRANK, M. 2013. Notes on the ecology of rolled-leaf hispines (Chrysomelidae, Cassidinae) at La Gamba (Costa Rica). ZooKeys 332: 55-69. https://doi.org/10.3897/ zookeys.332.5215

STAINES, C. 1996. The genus Cephaloleia (Coleoptera: Chrysomelidae) in Central America and the West Indies. Revista de Biología Tropical (Tomo especial) 3: 87.

STAINES, C. 1998. A new species of Cephaloleia Chevrolat (Coleoptera: Chrysomelidae: Hispinae) from Costa Rica. Proceedings of the Washington Entomological Society 100: 672673.

STAINES, C. 2008. A new species of Cephaloleia Chevrolat, 1837 (Coleoptera: Chrysomelidae: Cassidinae) from Dominica. Insecta Mundi 3 (3): 1-4.

STAINES, C.; GARCÍA-ROBLEDO, C. 2014. The genus Cephaloleia Chevrolat, 1836 (Coleoptera, Chrysomelidae, Cassidinae). Zookeys 436: 1-355. https://doi.org/10.3897/ zookeys. 436.5766

URUETA, E. 1972. Cephaloleia sp. cerca a vagelineata Pic una plaga de la palma africana. Revista Facultad Nacional de Agronomía 27 (2): 75-77.
URUETA, E. 1975. Insectos asociados con el cultivo de palma africana en Urabá (Antioquia) y estudio de su relación con la pudrición de la flecha - pudrición de cogollo. Revista Colombiana de Entomología 1 (4): 15-31.

\section{Origen y financiación}

El origen de los datos del presente artículo corresponde a un proyecto de investigación desarrollado por la Corporación Centro de Investigación en Palma de Aceite - Cenipalma y fue financiado por el Fondo de Fomento Palmero.

\section{Contribuciones de los autores}

Todos los autores contribuyeron en mayor o menor medida en la elaboración del presente articulo y sus contribuciones puntuales son las siguientes:

Carlos E. Barrios: Registro de datos en campo, montaje y conservación de los especímenes colectados, análisis de la información.

Natalia J. Castillo: Registro de datos en campo y laboratorio.

Alex E. Bustillo: Coordinación general del proyecto, revisión y corrección general del manuscrito de este artículo.

Charles L. Staines: Identificación taxonómica de las especies observadas. 